\title{
Use of standardized brief geriatric evaluation compared with routine care in general practice for preventing functional decline: a pragmatic cluster-randomized trial
}

\author{
Yolanda Mueller MD PhD, Joëlle Schwarz Msc PhD, Stéfanie Monod MD, Isabella Locatelli Msc PhD, \\ Nicolas Senn MD PhD
}

Cite as: CMAJ 2021 August 23;193:E1289-99. doi: 10.1503/cmaj.202887

\begin{abstract}
Background: Although assessment of geriatric syndromes is increasingly encouraged in older adults, little evidence exists to support its systematic use by general practitioners (GPs). The aim of this study was to determine whether a systematic geriatric evaluation performed by GPs can prevent functional decline.
\end{abstract}

Methods: We conducted a controlled, open-label, pragmatic cluster-randomized trial in 42 general practices in Switzerland. Participating GPs were expected to enrol an average of 10 communitydwelling adults (aged $\geq 75 \mathrm{yr}$ ) who understood French, and had visited their GP at least twice in the previous year. The intervention consisted of yearly assessment by the GP of 8 geriat- ric syndromes with an associated tailored management plan according to assessment results, compared with routine care. Our primary outcomes were the proportion of patients who lost at least 1 instrumental activity of daily living ( $A D L)$ and the proportion who lost at least 1 basic ADL, over 2 years. Our secondary outcomes were quality-of-life scores, measured using the older adult module of the World Health Organization Quality of Life Instrument, and health care use.

Results: Forty-two GPs recruited 429 participants (63\% women) with a mean age of 82.5 years (standard deviation $4.8 \mathrm{yr}$ ) at time of recruitment. Of these, we randomly assigned 217 participants to the intervention and 212 to the control arm. The proportion of patients who lost at least 1 instrumental ADL in the intervention and control arms during the course of the study was $43.6 \%$ and $47.6 \%$, respectively (risk difference $-4.0 \%, 95 \%$ confidence interval [Cl] $-14.9 \%$ to $6.7 \%, p=0.5)$. The proportion of patients who lost at least 1 basic ADL was $12.4 \%$ in the intervention arm and $16.9 \%$ in the control arm (risk difference $-5.1 \%, 95 \% \mathrm{Cl}-14.3 \%$ to $4.1 \%, p=0.3)$.

Interpretation: A yearly geriatric evaluation with an associated management plan, conducted systematically in GP practices, does not significantly lessen functional decline among communitydwelling, older adult patients, compared with routine care. Trial registration: ClinicalTrials.gov, NCT02618291.
$\mathrm{T}$ he World Health Organization has defined healthy aging as the process of developing and maintaining functional ability that enables well-being in older age. ${ }^{1}$ Functional ability is often measured by an individual's ability to perform activities of daily living (ADLs) without assistance. Geriatric syndromes, corresponding to multifactorial, chronic conditions, can impair physical and mental capacities, ${ }^{2-4}$ and are directly associated with functional decline. ${ }^{5}$ If recognized early, adapted preventive measures and management strategies can be started to limit functional decline. ${ }^{6-8}$ Interventions that have been shown to delay functional decline include comprehensive geriatric assessment, regular home visits and physical therapy. ${ }^{6,8,9} \mathrm{Com}$ prehensive geriatric assessment consists of a "multidisciplinary diagnostic and treatment process that identifies medical, psychosocial, and functional capabilities of older adults to develop a coordinated plan to maximize overall health with ageing." ${ }^{10}$ These assessments are usually performed by specialized geriatric teams for patients who have already been identified as frail or in the context of rehabilitation. However, most older patients see only their general practitioner (GP) and are not provided a comprehensive geriatric assessment, considering that this is a lengthy process that is often beyond the scope of a usual primary 
care consultation. A recent systematic review of comprehensive geriatric assessment in primary care found only 4 studies conducted in this setting, ${ }^{11}$ showing mixed effects on clinical outcomes. Only 1 study assessed functional ability, and it showed no impact in this context. ${ }^{12}$

In primary care, it may be more beneficial to use shorter screening tools. ${ }^{13-19}$ Previous studies using shorter tools adapted for primary care have failed to show a difference for patients compared with routine care. ${ }^{17,18}$ These interventions usually targeted patients who were already identified as frail or with a predefined number of problems. ${ }^{17,18,20}$

In contrast, our Active Geriatric Evaluation (AGE) tool targets all patients aged 75 and older. This clinical tool can be easily integrated to clinical encounters in GP practices, without the need for additional organizational changes. For this study, we aimed to determine whether the AGE tool, specifically designed for GPs and consisting of a brief assessment of the most relevant geriatric syndromes combined with management plans, could slow functional decline in older patients.

\section{Methods}

\section{Study design}

We conducted a controlled, open-label, pragmatic clusterrandomized trial in GP practices in western Switzerland. We used the PRECIS-2 criteria in designing the study to optimize direct applicability to GP practices. The study protocol is available at https://clinicaltrials.gov/ProvidedDocs/91/ NCT02618291/Prot_001.pdf.

\section{Participants}

We planned to recruit at least $40 \mathrm{GPs}$ (20 per arm), and each GP was expected to recruit 10 patients, on average. The GPs were recruited via letters sent by mail, professional societies' newsletters or personal contact by email or telephone. Participating GPs had to work at least 20 hours per week as GPs in French-speaking Switzerland. Only 1 GP per practice could participate to limit contamination. We excluded specialists in geriatrics and GPS who had participated in the validation study of the AGE tool.

We included patients if they were at least 75 years old at recruitment, living at home, able to understand French and had visited their GP at least twice in the previous year. We excluded patients who had had a geriatric or specialized memory consultation in the 3 months before recruitment, or who were planning to leave the study area or change GP in the next 2 years. Patients were enrolled by GPs before GP group assignment was revealed. The GPs could choose between assessing every patient aged at least 75 years for study eligibility until the target number of inclusion was reached, or proposing the study only to a random sample of their patients. In this latter scenario, we asked GPs to decide on a fixed number of patients to be included per day ( 1 or 2 ), selected from the pre-eligible patients on the day's agenda using a list of random numbers. Written consent was sought by GPs from individual participants before randomization.

\section{Setting}

Most Swiss GP practices are small, self-owned practices with 2-4 GPs. Integrated nurse practitioners and social workers are uncommon. Community-based services, such as home-based care, physical or occupational therapy are prescribed by GPs, but delivered outside of GP practices.

\section{Randomization and blinding}

The randomization unit was the GP, with GPs assigned on a 1:1 ratio to the intervention or usual care arm. An independent researcher generated a computer-based randomization list, using uneven block sizes. She then prepared sealed, opaque envelopes containing information on the treatment arm, with a printed identification number on the outside. During training sessions that took place after patient enrolment, GPs were assigned to their respective arm upon opening the envelope corresponding to their predefined unique identification number.

The research assistant performing the main outcome measures (telephone interviews), study coordinator and study statistician were blinded to the randomization. Participants, GPS and study assistants who conducted the annual visits to the family practice were unblinded to the GP's assignment. Specific sections of the electronic case report form, which revealed assignment, were coded so that blinded staff could not link these data to participant or GP identifiers.

\section{Intervention}

The intervention comprised a yearly, brief assessment, guided by the AGE tool (Figure 1) of 4 ADLs (3 instrumental ADL and 1 basic $A D L$ ) and screening for 8 geriatric syndromes, namely cognitive impairment, mood disorder, gait and balance impairment, visual impairment, hearing impairment, urinary incontinence, malnutrition and osteoporosis (Table 1), followed by proposal of a management plan based on the results of the evaluation. Details of the construction and validation of the AGE tool have been published elsewhere..$^{15,21,22}$

The suggestions for management were divided into 2 distinct steps: additional tests after a positive screen to confirm or exclude diagnoses, and specific management approaches. All suggested approaches were based on a literature review ${ }^{15}$ and geriatrician expertise. Management suggestions were further graded as major and minor. To preserve the pragmatic approach of the study, GPs were free to implement the proposed suggestions and approaches.

The AGE tool was available either as an electronic tool (embedded in the case report form) or as a paper summary. In the electronic form, sections of the management plan relative to specific geriatric syndromes appeared if selected in the screening process. In addition, GPs could add or delete specific sections of the management plan. We intended for the tool to be administered by GPs, but GPs could delegate the screening to medical assistants. General practitioners assigned to the intervention arm received a 2-hour, face-to-face, small group training session on the AGE tool from an academic GP and a geriatrician, and received a reference book on comprehensive 


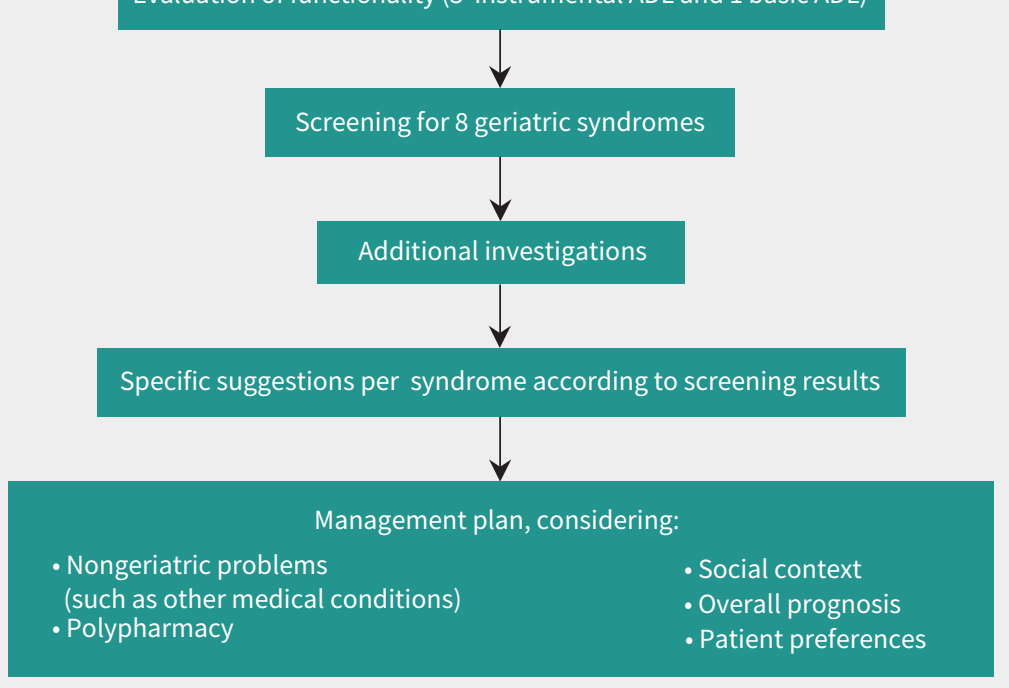

Figure 1: Conceptual framework of the Active Geriatric Evaluation (AGE) tool. Note: ADL = activity of daily living. Adapted from Sen and Monod (licensed under CC BY 4.0)..$^{15}$

geriatric assessment. ${ }^{23}$ The intervention was delivered during routine face-to-face consultations. If a single consultation was not sufficient, it could be spread over multiple consultations. Both GP groups received a 1-hour training session on study procedures. General practitioners of the control group received no specific training related to the study intervention.

General practitioners integrated follow-up patient assessments into regular consultations, with a final outcome visit encouraged after 2 years, plus a 3-month window. A research assistant conducted annual reviews of medical records in the practice, extracting data on the number of consultations and content (e.g., type of clinical examination, weight and height measures, evaluations of alcohol consumption, nutrition and physicial exercise), laboratory tests, radiological evaluations, new diagnoses of chronic conditions (coded using the International Classification of Primary Care, version 2), medications, specialist referrals, emergency consultations and hospital admissions. In parallel, a different research assistant conducted annual telephone interviews to assess patientreported outcomes.

\section{Outcomes}

The primary outcomes were the proportion of patients who lost independence in at least 1 instrumental $A D L$ and the proportion of patients who lost independence in at least 1 basic ADL over 2 years. We scored ADLs as 0 or $1,{ }^{21}$ considering 8 instrumental ADLs (i.e., using the telephone, shopping, food preparation, housekeeping, laundry, mode of transportation, responsibility for own medications and ability to handle financing ${ }^{24}$ and 6 basic ADLs (i.e., bathing, dressing, toileting, transferring, continence and feeding). ${ }^{25}$ These 2 measures have been used in numerous studies because of their robust psychometric properties, their sensitivity to change, their simplicity, and the fact that they can be reliably evaluated over the telephone. ${ }^{26,27}$ According to previous studies that used this outcome in similar populations, avoiding a 1-point (i.e., 1 activity) loss out of 8 activities (for instrumental ADLs) can be considered clinically meaningful.,28 We decided against treating the outcome as a continuous variable (and comparing the mean difference between the 2 arms) after preliminary analysis of baseline data showed that most patients had a baseline instrumental ADL score of 8 (maximal score).

Secondary outcomes included mean quality-of-life score, measured using the older adult module of the World Health Organization Quality of Life Instrument (WHOQOL-OLD), and incidence of hospital admissions, institutionalizations, emergency department visits and outpatient visits. We also compared the 2 groups in terms of the number of geriatric syndromes identified and the adopted management strategies, such as medication adaptation, referral to specialty care or supportive measures.

We asked GPs to record any serious adverse events (e.g., hospitalization, death) in the electronic case report form within 7 days of their occurrence. For each serious adverse event, GPs collected the time of onset, duration, resolution, action to be taken, assessment of intensity and relation to the study intervention. For every step of the management plan, GPs recorded whether they adhered to the suggestion or not. We estimated GP adherence to the intervention at each step of the AGE tool. In the study protocol, we prespecified quantitative outcomes of acceptability (i.e., proportion of GPs using 


\begin{tabular}{|c|c|c|c|}
\hline Syndrome & Screening & $\begin{array}{l}\text { Additional investigation if screening } \\
\text { positive (diagnostic confirmation)* }\end{array}$ & Proposed management approaches ${ }^{\star}$ \\
\hline Functionality & $\begin{array}{l}4 \text { questions: Can you dress yourself? } \\
\text { Can you prepare your meals alone? Can } \\
\text { you do your own shopping? Can you } \\
\text { make your payments alone? }\end{array}$ & & \\
\hline $\begin{array}{l}\text { Urinary } \\
\text { incontinence }\end{array}$ & $\begin{array}{l}4 \text { questions: Do you have difficulty } \\
\text { holding in your urine or feel urge to } \\
\text { urinate? Do you sometimes find it } \\
\text { difficult to reach the toilet in time? Do } \\
\text { you have involuntary urine loss when } \\
\text { coughing or on effort? Do you } \\
\text { sometimes wear protection pads? }\end{array}$ & $\begin{array}{l}\text { Complete focused medical history } \\
\text { and examination: sensation of } \\
\text { emptying, dysuria, pollakiuria, } \\
\text { urogynecological problems, urinary } \\
\text { retention, prolapse, rectal } \\
\text { examination } \\
\text { - Voiding calendar (timing of } \\
\text { urination, nocturia) } \\
\text { - Targeted clinical examination (e.g., } \\
\text { urinary retention, prolapse, rectal } \\
\text { examination) } \\
\text { - Urinary dipstick } \\
\text { - Radiological evaluation for } \\
\text { posturination residue } \\
\text { - Review medication }\end{array}$ & $\begin{array}{l}\text { - Prescribe urinary protection pads } \\
\text { - Consider specialized physiotherapy } \\
\text { and rehabilitation } \\
\text { - Discuss voiding behavioural hygiene } \\
\text { - Consider anticholinergic or a-blocker } \\
\text { - Refer to gynecologist or urologist for } \\
\text { specialty care (e.g., surgery) }\end{array}$ \\
\hline Mood disorder & PHQ-2 & $\begin{array}{l}\text { - Complete medical history } \\
\text { - Perform Geriatric Depression Scale } \\
\text { (short form) } \\
\text { - Assess alcohol consumption }\end{array}$ & $\begin{array}{l}\text { - Initiate depression follow-up } \\
\text { - Prescribe antidepressant drug } \\
\text { - Conduct motivational intervention } \\
\text { on alcohol consumption }\end{array}$ \\
\hline $\begin{array}{l}\text { Cognitive } \\
\text { impairment }\end{array}$ & Mini-Cog & 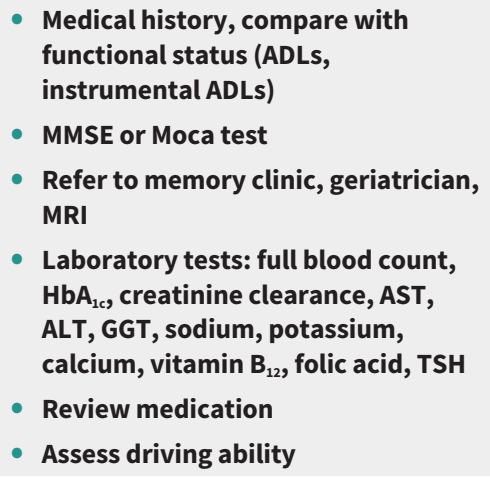 & $\begin{array}{l}\text { Refer for home care support } \\
\text { - Meet family/network } \\
\text { Consider specific treatment } \\
\text { (hypothyroidism) } \\
\text { - Prescribe acetylcholinesterase } \\
\text { inhibitors } \\
\text { - Adapt medication }\end{array}$ \\
\hline $\begin{array}{l}\text { Visual } \\
\text { impairment }\end{array}$ & Near vision pocket card & $\begin{array}{l}\text { Complete visual acuity assessment } \\
\text { (Snellen chart) } \\
\text { Refer to ophthalmologist for full } \\
\text { assessment (e.g., cataracts, } \\
\text { glaucoma) }\end{array}$ & $\begin{array}{l}\text { Refer to occupational therapist to } \\
\text { check indication for auxiliary means }\end{array}$ \\
\hline $\begin{array}{l}\text { Hearing } \\
\text { impairment }\end{array}$ & Whisper test & $\begin{array}{l}\text { Perform otoscopy (cerumen } \\
\text { impaction) } \\
\text { - Refer for audiometry }\end{array}$ & - Prescribe hearing aid \\
\hline
\end{tabular}

the tool once or twice during the study follow-up) and feasibility (i.e., physician adherence to management sugggestions). We evaluated these outcomes in combination with our qualitative assessment of acceptability and feasibility, as well as perceptions of autonomy, assessed by semistructured interviews of a subset of patients and GPs. The results from the qualitative component of this research have been presented elsewhere. ${ }^{29}$

\section{Statistical analysis}

To estimate the sample size, we assumed that $10 \%$ of patients would lose independence in at least 1 instrumental ADL in the intervention arm and $25 \%$ in the control arm. These proportions were based on previous trials ${ }^{6-8}$ and longitudinal studies. ${ }^{3}$ Using these parameters, we generated cluster data with various combinations of the number of GPs per arm and patients per GP for different levels of intraclass correlation 


\begin{tabular}{|c|c|c|c|}
\hline Syndrome & Screening & $\begin{array}{l}\text { Additional investigation if screening } \\
\text { positive (diagnostic confirmation)* }\end{array}$ & Proposed management approaches* \\
\hline \multirow[t]{2}{*}{$\begin{array}{l}\text { Gait and } \\
\text { balance }\end{array}$} & History of falls during past year & $\begin{array}{l}\text { Complete medical history and } \\
\text { examination: cardiovascular, } \\
\text { neurologic, osteoarticular, } \\
\text { Schellong test } \\
\text { Examine feet and shoes }\end{array}$ & $\begin{array}{l}\text { Refer for home hazard assessment } \\
\text { (occupational therapist) and home } \\
\text { care support }\end{array}$ \\
\hline & Gait observation & $\begin{array}{l}\text { Refer to specialty care if needed } \\
\text { (e.g., neurology) } \\
\text { - Review medication } \\
\text { Assess alcohol consumption } \\
\text { - Check calcium and vitamin D }\end{array}$ & $\begin{array}{l}\text { Prescribe exercise, physiotherapy, } \\
\text { adapted shoes } \\
\text { - Adapt medication } \\
\text { - Conduct motivational intervention } \\
\text { on alcohol consumption } \\
\text { - Consider calcium and vitamin D } \\
\text { prescription }\end{array}$ \\
\hline Osteoporosis & $\begin{array}{l}\text { - History of osteoporotic fracture } \\
\text { - Height loss since age } 25 \\
\text { - Occiput-wall and rib-pelvis distance }\end{array}$ & $\begin{array}{l}\text { - Perform osteodensitometry } \\
\text { - Check calcium and vitamin D }\end{array}$ & $\begin{array}{l}\text { - Prescribe exercise, physiotherapy } \\
\text { Consider calcium and vitamin D } \\
\text { supplementation } \\
\text { - Consider treatment with } \\
\text { biphosphonates }\end{array}$ \\
\hline Undernutrition & $\begin{array}{l}\text { Weight loss }>5 \% \text { past month or } 10 \% \\
\text { past } 6 \text { months }\end{array}$ & $\begin{array}{l}\text { Perform digestive (including } \\
\text { constipation) and dental } \\
\text { examination } \\
\text { - Review medication } \\
\text { - Assess financial situation }\end{array}$ & $\begin{array}{l}\text { - Treat other causes (e.g., depression } \\
\text { - Refer for home care support (e.g., } \\
\text { meals, shopping) } \\
\text { - Prescribe hyperproteic supplements }\end{array}$ \\
\hline
\end{tabular}

coefficient (ICC). To achieve a power of 90\%, 8 patients per GP would provide a sufficient sample size if we had 20 GPs per arm, based on an ICC of 0.10 . Assuming a loss to follow-up of $15 \%$, we increased the number of patients per GP to $8 /(1-0.15)$ $=10$, corresponding to a final sample size of $40 \mathrm{GPs}$ with a total of 400 patients.

For our primary analysis, we compared the proportions of patients in the intervention and control groups who had lost at least 1 instrumental ADL and those who had lost at least 1 basic ADL after 2 years, using a generalized logistic mixed-effect model, including a random effect for the physician.

For our secondary analyses, prespecified in the statistical analysis plan, we compared the mean reduction in WHOQOLOLD score after 2 years between the intervention and control arms using a generalized linear mixed-effect model. We also compared the proportions of patients with hospital admissions, institutionalizations and emergency department visits by arm, as well as the number of routine visits, and the time to institutionalization or death. All comparisons between treatment arms used mixed models that included a random effect for cluster. We used mixed-effect negative binomial regression to compare the number of GP consultations, specialist consultations and patient weight measurements, and mixed-effect logistic regression for binary secondary outcomes (i.e., patients with at least 1 emergency consultation, hospital admission, stay in an institution, new chronic condition diagnosis, severe adverse event, communication between GP and home-based care, communication between GP and the family and presence of polymedication). We adopted Cox survival analysis techniques to compare time to institutionalization or death. We used longitudinal models, including a second random effect for a participant's repeated measures, to estimate the 2-year evolution of the mean number of instrumental ADLs and basic ADLs (2-level, mixed-effect Poisson models) and mean WHOQOL-OLD scores (2-level, mixed-effect linear model).

All patients recruited by the randomized GPs were included in the analysis. The primary analysis was a complete case analysis that included all patients with instrumental or basic ADLs measured at baseline and after 2 years. After complete case analysis, we performed prespecified sensitivity analyses with the last observation carried forward, and considering patients who had died or who were institutionalized as having lost 1 instrumental ADL (full analysis set). We performed an additional sensitivity analysis that included all patients with baseline outcome assessment, where all those missing followup assessment were considered as having lost 1 instrumental 


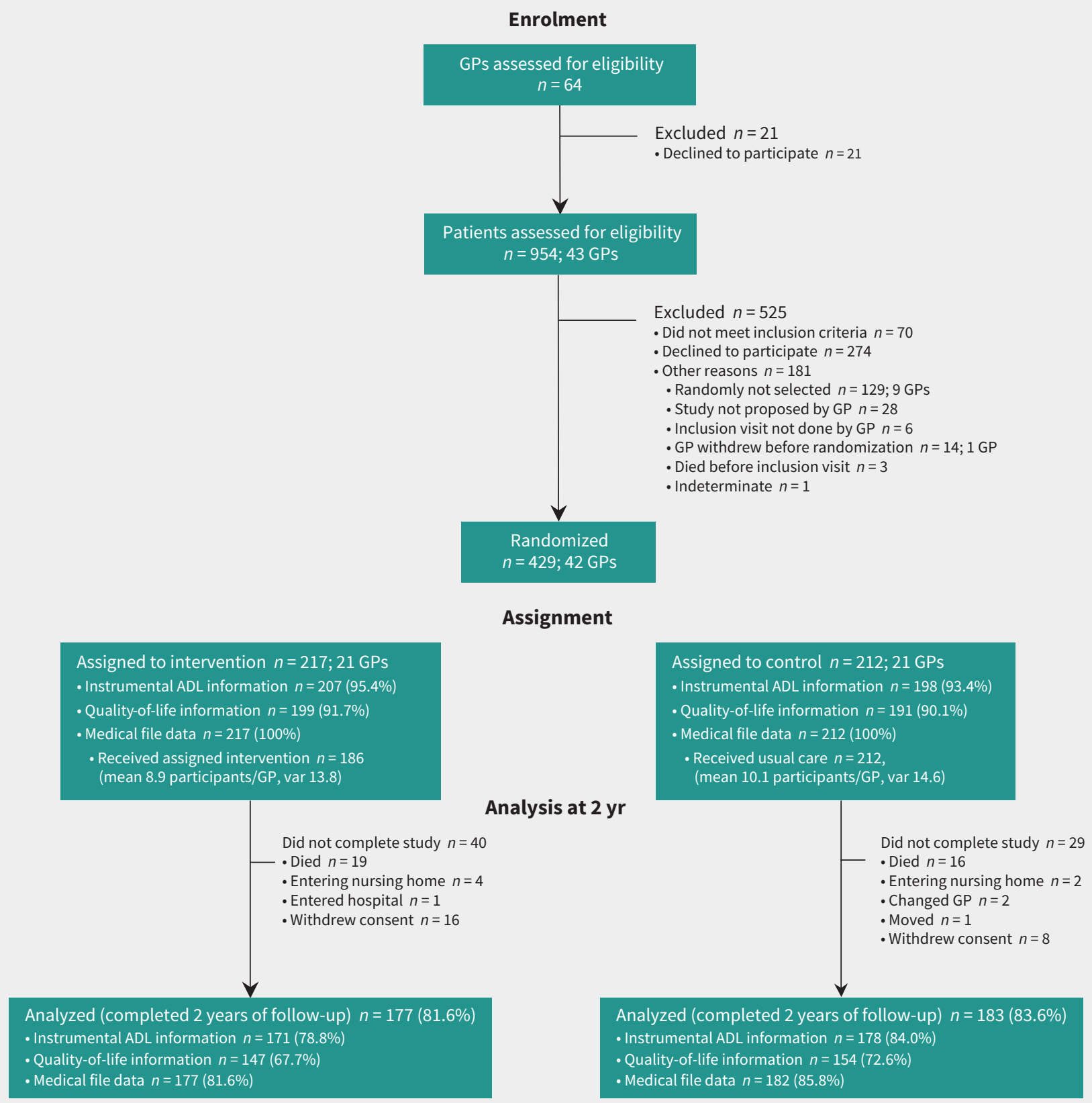

Figure 2: Study flowchart. Note: $A D L=$ activity of daily living, $G P=$ general practitioner, var = variance.

or basic ADL. We also analyzed the per-protocol population, excluding participants in the intervention arm who received fewer than 2 complete screenings (i.e., at least 7 out of 8 items screened) or participants for whom GPs followed fewer than half of the proposed major suggestions.

We conducted analyses in $\mathrm{R}$ version 3.5, and Stata version 16.1. The steering committee, composed of all investigators, reviewed the planned interim analysis of primary and secondary outcomes when at least $50 \%$ of patients had their 1-year assessment, and reviewed the safety analysis on serious adverse events.

\section{Ethics approval}

This study was approved by the cantonal ethics committee on May 30, 2016 (CER-VD no. 2016-00422). The study followed the World Medical Association's Declaration of Helsinki. Participants gave written informed consent before taking part.

\section{Results}

We recruited 42 GPs to participate in the study. Of 954 patients screened for eligibility between Aug. 12, 2016, 
Table 2: Baseline patient characteristics

\begin{tabular}{|c|c|c|c|}
\hline \multirow[b]{2}{*}{ Characteristic } & \multicolumn{3}{|c|}{$\begin{array}{l}\text { No. }(\%) \text { of } \\
\text { patients* }\end{array}$} \\
\hline & $\begin{array}{c}\text { Total } \\
n=429\end{array}$ & $\begin{array}{c}\text { Intervention } \\
n=\mathbf{2 1 7}\end{array}$ & $\begin{array}{l}\text { Control } \\
n=212\end{array}$ \\
\hline Sex, female & $269(62.7)$ & $141(65.0)$ & $128(60.4)$ \\
\hline $\begin{array}{l}\text { Age at recruitment, } \\
y r, \text { mean } \pm S D\end{array}$ & $82.5 \pm 4.8$ & $82.7 \pm 4.7$ & $82.4 \pm 4.9$ \\
\hline \multicolumn{4}{|l|}{ Equipment $†$} \\
\hline Glasses & $325(75.8)$ & $184(84.8)$ & $141(66.5)$ \\
\hline Hearing aids & $105(24.5)$ & $53(24.4)$ & $52(24.5)$ \\
\hline Urinary protections & $93(21.7)$ & $51(23.5)$ & $42(19.8)$ \\
\hline Dentures & $221(51.5)$ & $110(50.7)$ & $111(52.4)$ \\
\hline \multicolumn{4}{|l|}{ Driving } \\
\hline Still driving & $219(51.1)$ & $108(49.8)$ & $111(52.4)$ \\
\hline Stopped driving & $99(23.1)$ & $47(21.7)$ & $52(24.5)$ \\
\hline Never drove & $91(21.2)$ & $50(23.0)$ & $41(19.3)$ \\
\hline Home-based care & $75(17.5)$ & $38(17.5)$ & $37(17.5)$ \\
\hline Nursing & $55(73.3)$ & $28(73.7)$ & $27(73.0)$ \\
\hline Meals & $19(25.3)$ & $12(31.6)$ & $7(18.9)$ \\
\hline Domestic help & $37(49.3)$ & $17(44.7)$ & $20(54.1)$ \\
\hline $\begin{array}{l}\text { Personal hygiene } \\
\text { and comfort }\end{array}$ & $28(37.3)$ & $12(31.6)$ & $16(43.2)$ \\
\hline Occupational therapy $\ddagger$ & $16(3.7)$ & $8(3.7)$ & $8(3.8)$ \\
\hline Physiotherapy $\ddagger$ & $134(3.2)$ & $67(30.9)$ & $67(31.6)$ \\
\hline \multicolumn{4}{|l|}{ Environment } \\
\hline $\begin{array}{l}\text { Lives on his or } \\
\text { her own }\end{array}$ & $215(50.1)$ & $121(55.8)$ & $94(44.3)$ \\
\hline Lives with partner & $197(45.9)$ & $90(41.5)$ & $107(50.5)$ \\
\hline $\begin{array}{l}\text { Lives with child or } \\
\text { other }\end{array}$ & $16(3.7)$ & $5(2.3)$ & $11(5.2)$ \\
\hline $\begin{array}{l}\text { No. of children, } \\
\text { median (IQR) }\end{array}$ & 2 (1 to 2 ) & 2 (1 to 2 ) & 2 (1 to 2 ) \\
\hline Other caregivers & $100(23.3)$ & $55(25.4)$ & $45(21.2)$ \\
\hline $\begin{array}{l}\text { No. of chronic } \\
\text { conditions, } \\
\text { median (IQR) }\end{array}$ & 4 (2 to 6$)$ & 4 (2 to 5$)$ & 4 (3 to 6$)$ \\
\hline $\begin{array}{l}\text { No. of medications, } \\
\text { median (IQR) }\end{array}$ & 5 (3 to 7$)$ & 5 (3 to 7$)$ & 5 (3 to 7.5$)$ \\
\hline \multicolumn{4}{|c|}{ Previous selected surgeries } \\
\hline Hip replacement & $69(16.1)$ & $34(15.7)$ & $35(16.5)$ \\
\hline Knee replacement & $54(12.6)$ & $25(11.5)$ & $29(13.7)$ \\
\hline Cataract surgery & $121(28.2)$ & $65(30.0)$ & $56(26.4)$ \\
\hline
\end{tabular}

Note: IQR = interquartile range, $\mathrm{SD}=$ standard deviation

*Unless indicated otherwise.

†Missing considered as 0 and included in denominator for proportions: 20 were missing for driving; 8 were missing for dentures; 3 for hearing aids, meals and other caregivers; 2 for urinary protections and personal hygiene and comfort; none or 1 for all other variables.

$\ddagger$ Number of patients with a prescription during past year. and Nov. 15, 2017, GPs recruited 429 patients between Sept. 26, 2016, and Jan. 29, 2018 (Figure 2), with follow-ups completed by Jan. 31, 2020. Among participating patients, $63 \%$ were female and the mean age was 82.5 years (standard deviation $4.8 \mathrm{yr}$ ). Baseline sociodemographic and clinical characteristics are shown in Table 2.

In the primary analysis, the proportion of patients who lost independence in at least 1 instrumental ADL during the course of the study was $43.6 \%$ and $47.7 \%$ in the intervention and control arms, respectively (risk difference $-4.0 \%, 95 \%$ confidence interval $[\mathrm{Cl}]-14.9 \%$ to $6.7 \%, p=0.5$ ) (Table 3 ). The proportion of patients who lost independence in at least 1 basic ADL was $12.4 \%$ and $16.9 \%$ in the intervention and control arms, respectively (risk difference $-5.1 \%, 95 \% \mathrm{Cl}-14.3 \%$ to $4.1 \%, p=0.3)$.

The mean reduction in quality-of-life scores in the intervention and control groups was -0.12 (SD 7.36) and 0.74 (SD 7.76), respectively $(p=0.3)$. We did not observe significant differences between study arms for any secondary outcomes assessed regarding health care use (i.e., number of consultations, emergency consultations, hospital admissions or institutional stays), type of health care (i.e., number of weight measures and number of specialists involved) or communication with home-based care or families (Table 4). Time to institutionalization or death was not different between patients receiving AGE or usual care by log-rank test $(p=0.27)$. The proportion of patients experiencing severe adverse events was not different between the treatment arms.

In the sensitivity analysis, considering death or admission to an institution as having lost at least 1 instrumental ADL increased the number of patients analyzed in the complete case analysis from 339 to 381 . We did not observe a significant difference between arms in the proportion of patients who had lost at least 1 instrumental ADL (50.0\% in the intervention and $51.8 \%$ in the control arms, respectively, $p=0.7, \mathrm{ICC}=0.00$ ) (Appendix 1, supplementary material, available at www.cmaj. ca/lookup/doi/10.1503/cmaj.202887/tab-related-content). Longitudinal analysis of instrumental ADL and basic ADL scores for the entire study population, and stratified by age and gender, can be found in Appendix 1.

Overall, of 179 patients with a baseline screening using the AGE tool, 119 (66.5\%) were screened again after 1 year. A median of 3 (interquartile range [IQR] 1 to 4 ) syndromes was suspected at the first visit, and a median of 2 (IQR 1 to 3 ) syndromes was suspected at the second visit. Overall, GPs adhered to $59.9 \%$ of all the major suggestions of the management plan. In the intervention group, GPs adhered to at least $50 \%$ of the suggested items for $48.2 \%$ of patients (details provided in the supplementary material, Appendix 1). Overall adherence, including both the assessment and management plan, was $61.6 \%$ (95\% Cl $48.5 \%$ to $74.7 \%$, adjusted for cluster). In the per-protocol population, which excluded 118 patients in the intervention arm with low GP adherence, we did not observe any significant differences between the intervention and control arms in the proportion of patients who had lost at least 1 instrumental ADL $(45.7 \% \mathrm{v}$. $47.6 \%, p=0.8, I C C=0.01)$, in the proportion of patients who 
Table 3: Proportion of patients who lost activities of daily living (ADLs) between baseline and 2 years assessment, according

to treatment arm

\begin{tabular}{|c|c|c|c|c|c|c|c|c|}
\hline \multirow[b]{2}{*}{ Variable } & \multicolumn{2}{|c|}{$\begin{array}{l}\text { No. of patients with ADL } \\
\text { information }\end{array}$} & \multicolumn{2}{|c|}{$\begin{array}{c}\text { No. }(\%) \text { of patients who lost } \\
\text { at least } 1 \mathrm{ADL}\end{array}$} & \multirow{2}{*}{$\begin{array}{l}\text { Estimated } \\
\text { difference } \\
(95 \% \mathrm{Cl})^{\star}\end{array}$} & \multirow[b]{2}{*}{ OR (95\%CI) } & \multirow{2}{*}{$\begin{array}{c}p \\
\text { value }\end{array}$} & \multirow{2}{*}{$\begin{array}{c}\text { ICC } \\
(95 \% \mathrm{Cl})\end{array}$} \\
\hline & Intervention & Control & Intervention & Control & & & & \\
\hline $\begin{array}{l}\text { Instrumental } \\
\mathrm{ADL}\end{array}$ & 165 & 174 & $72(43.6)$ & $83(47.7)$ & $-4.0(-14.9$ to 6.7$)$ & $\begin{array}{c}0.85(0.53 \text { to } \\
1.35)\end{array}$ & 0.5 & $\begin{array}{l}0.012(0.001 \\
\text { to } 0.107)\end{array}$ \\
\hline Basic ADL & 169 & 178 & $21(12.4)$ & 30 (16.9) & $-5.1(-14.3$ to 4.1$)$ & $\begin{array}{c}0.64 \text { ( } 0.26 \text { to } \\
1.41)\end{array}$ & 0.3 & $\begin{array}{l}0.139(0.048 \\
\text { to } 0.341)\end{array}$ \\
\hline
\end{tabular}

Table 4: Secondary and safety outcomes, according to treatment arm

\begin{tabular}{|c|c|c|c|c|c|}
\hline \multirow[b]{2}{*}{ Variable } & \multicolumn{2}{|c|}{ No. $(\%)$ of patients* } & \multirow[b]{2}{*}{ IRR/OR/HR† } & \multirow[b]{2}{*}{$95 \% \mathrm{Cl}$} & \multirow[b]{2}{*}{$p$ value } \\
\hline & $\begin{array}{c}\text { Intervention } \\
\quad n=\mathbf{2 1 7}\end{array}$ & $\begin{array}{l}\text { Control } \\
n=212\end{array}$ & & & \\
\hline GP consultations, median (IQR) & 15.0 (9.4 to 22.0$)$ & 16.0 (10.0 to 22.0$)$ & 0.93 & 0.79 to 1.10 & 0.40 \\
\hline No. of patient weight measures, median (IQR) & $3(1$ to 5$)$ & 3 (1 to 6 ) & 0.95 & 0.60 to 1.49 & 0.82 \\
\hline At least 1 stay in institution & $11(5.1)$ & $10(4.7)$ & 0.74 & 0.42 to 1.29 & 0.28 \\
\hline At least 1 new chronic condition diagnosed $\ddagger$ & $58(28.9)$ & $57(28.9)$ & 0.95 & 0.43 to 2.09 & 0.89 \\
\hline Polymedication ( $>4$ drugs) at $2 \mathrm{yr}$ & $115(65.0)$ & $121(66.1)$ & 0.94 & 0.59 to 1.50 & 0.80 \\
\hline Potentially inappropriate medication§ & $133(75.1)$ & $153(83.6)$ & 0.60 & 0.30 to 1.19 & 0.14 \\
\hline Occurrence of any severe adverse event & $61(28.1)$ & $67(31.6)$ & 1.55 & 0.68 to 3.51 & 0.30 \\
\hline Time to death or to admission to an institution & - & - & 1.43 & 0.76 to 2.72 & 0.27 \\
\hline \multicolumn{6}{|c|}{$\begin{array}{l}\text { Note: } \mathrm{Cl}=\text { confidence interval, } \mathrm{GP}=\text { general practitioner, } \mathrm{HR}=\text { hazard ratio, } \mathrm{IQR}=\text { interquartile range, IRR = incidence rate ratio, } \mathrm{OR}=\text { odds ratio. } \\
\text { *Unless indicated otherwise. } \\
\text { †IRRs are based on mixed negative binomial regression model, adjusted for cluster effect at } \mathrm{GP} \text { level. ORs (for binary outcomes) are based on mixed logistic regression model, adjusted } \\
\text { for cluster effect at } \mathrm{GP} \text { level. } \mathrm{HR} \text { is based on Cox survival analysis for time to death or admission to an institution. } \\
\text { †Based on a selection of codes from the International Classification for Primary Care, version } 2 \text {. } \\
\text { \$Potentially inappropriate medication according to American Geriatrics Society } 2015 \text { updated Beers criteria. }\end{array}$} \\
\hline
\end{tabular}

had lost at least 1 basic ADL ( $10.9 \%$ v. $16.6 \%, p=0.3$, ICC $=0.10)$ or in the WHOQOL-OLD score after 2 years $(-0.32$ v. $0.71, p=0.4$, ICC $=0.01$ ) (Appendix 1).

\section{Interpretation}

Systematic screening for and management of geriatric syndromes in general practice using the AGE tool (which screens for most of the items used in similar tools ${ }^{30}$ and covers all areas recommended by WHO's approach to integrated care for older people [ICOPE] ${ }^{19}$ ) did not slow functional decline of patients aged 75 years and older over a 2-year course compared with routine care. The intervention also showed no effect on quality-of-life scores or health care use. In our study, the intervention was a brief assessment delivered by GPs, rather than a comprehensive geriatric assessment delivered by geriatricians or specifically trained nurse practitioners, as is often used in other trials. Our results differ from those of studies that used home-based management programs, which have shown a positive effect in preventing functional decline in participants..$^{8,9,31}$ Other studies using shorter tools adapted for GPs have also failed to show an impact on functional decline. ${ }^{17,18}$ These interventions targeted patients identified as frail or with a predefined number of problems, whereas our study included all patients aged 75 and older. We observed higher functional decline than anticipated in our study participants, as well as a higher-than-anticipated proportion of 
deaths, which, we think, highlights the importance of targeting all older adults with such interventions.

Several reasons may explain the absence of effect of the intervention. First, unlike trials targeting underserved populations, ${ }^{31}$ usual care in the Swiss context may already be very good, as shown by the high proportion of patients already equipped with hearing aids or having undergone cataract surgery at baseline. Second, the intervention was of moderate intensity and pragmatic nature, which may have lessened its impact. A more controlled implementation of all items of the management plan may have allowed us to detect a difference in patient outcomes. However, the fact that we did not observe a difference in the per-protocol population does not support the hypothesis of insufficient adhesion. Also, as further investigations or interventions were negotiated with patients in the long term, they may not have been captured within the trial's timeframe.

Although a substantial body of evidence describes the processes and predictors of functional decline, few interventions have successfully modified individual life-course trajectories. The question of when and in what population to begin interventions is still unresolved. Evidence from the Whitehall II study suggests that prevention of frailty should begin in midlife, ${ }^{32}$ but how and when to promote this patient-provider dialogue should be the objective of future research.

Despite our negative findings, we would argue that GP practices are the right setting to guide patients in aging because of their wide population coverage. However, interprofessional teams, rather than physicians alone, may better deliver this care, which should also include assessment of patients' goals. In Switzerland, most physicans in primary care work alone and rarely in multprofessional teams; ${ }^{33}$ however, other jurisdictions, such as the United Kingdom, the Netherlands or Canada, are more familiar with integrated practices, with physicians, nurses and other health care professionnals working together. It is likely that such settings, with stronger interprofessional collaborations and better organization of care delivery at the system level, may allow for consolidated patient management strategies and better functional outcomes, even though GPs continue to play a key role in multidisciplinary teams. ${ }^{34,35}$

\section{Limitations}

One area of concern is bias due to deviation from the intended intervention. Indeed, GPs in the control arm may have been more attentive to the functional issues of their older patients because of their participation in the study. Furthermore, GPs in the intervention arm did not adhere to all suggestions of the tool, which was consistent with the pragmatic nature of the trial. In terms of external validity, a certain amount of selection is unavoidable when conducting trials that involve a substantial investment from participating GPs. Participating GPs may have been more interested in geriatric care and be more up-to-date with continuing education in this area. Thus, practices in the control arm may have provided better care than average practices. In addition, GP practices in Switzerland are very physician-centred. Our study results may not be valid in different primary care settings where interprofessional teams could have enhanced the impact of the intervention.

Our patient recruitment method may have favoured slightly younger patients (Appendix 1, Table S1) and may have excluded the most vulnerable patients. Apart from this, we did not observe important baseline differences between patients in the intervention and control groups. Loss to follow-up was within the expected range and comparable between groups. The main area for concern was missingness of certain outcomes, such as ADL or quality-oflife scores, where missingness was potentially dependent on the score value. However, the sensitivity analysis in which we considered missing outcomes as negative is reassuring on this point. Finally, our assumptions regarding loss of independence that guided the sample size estimation were overly optimistic. We did not adjust expected rate of functional decline or $p$ values to the fact that we had 2 primary outcomes; we calculated sample sizes using only expected rates of instrumental ADL loss, not basic ADL loss. As our findings were negative, however, this does not affect the general thrust in the results.

Our choice of outcomes could be criticized. First, the use of a disability criterion in instrumental ADLs causes a number of methodological problems (i.e., choice of items and categories, ceiling effect, lack of gender sensitivity). ${ }^{36}$ Second, many study participants found the WHOQOL-OLD to be intrusive, resulting in some participant withdrawals or incomplete data. Few clinical chronic care interventions have actually been able to improve patient quality of life. ${ }^{37}$ We did not include patient-centred measures, such as measures of patient satisfaction or continuity of care, ${ }^{38-40}$ as our intervention did not target patient-centredness or integrated care per se. The AGE intervention did not explicitly elicit patient goals. More recent approaches that promote goaloriented care and assess individual goal attainment are promising. ${ }^{41-43}$ On the other hand, studies in the field of multimorbidity have shown contrasting results on quality of life, even if a goaloriented, patient-centred approach was used. ${ }^{37}$

\section{Conclusion}

Encouraging GPs to screen older patients for geriatric syndromes using a brief assessment of activities of daily living and to propose management approaches is not sufficient to slow the functional decline of patients aged 75 and older. Systemlevel interventions may be needed to promote integrated care that includes patient preferences.

\section{References}

1. World report on ageing and health. Geneva: World Health Organization; 2015.

2. Monod-Zorzi S, Seematter-Bagnoud L, Büla C, et al. Maladies chroniques et dépendance fonctionnelle des personnes âgées: données épidémiologiques et économiques de la littérature. Neuchâtel (Switzerland): Observatoire suisse de la santé; 2007.

3. Spiers NA, Matthews RJ, Jagger C, et al. Diseases and impairments as risk factors for onset of disability in the older population in England and Wales: findings from the Medical Research Council Cognitive Function and Ageing Study. J Gerontol A Biol Sci Med Sci 2005;60:248-54. 
4. Tinetti ME, Inouye SK, Gill TM, et al. Shared risk factors for falls, incontinence, and functional dependence. Unifying the approach to geriatric syndromes. JAMA 1995;273:1348-53.

5. Rosso AL, Eaton CB, Wallace R, et al. Geriatric syndromes and incident disability in older women: results from the women's health initiative observational study. J Am Geriatr Soc 2013;61:371-9.

6. Stuck AE, Aronow HU, Steiner A, et al. A trial of annual in-home comprehensive geriatric assessments for elderly people living in the community. N Engl J Med 1995;333:1184-9.

7. Tinetti ME, Baker DI, McAvay G, et al. A multifactorial intervention to reduce the risk of falling among elderly people living in the community. $N$ Engl J Med 1994;331:821-7.

8. Gill TM, Baker DI, Gottschalk M, et al. A program to prevent functional decline in physically frail, elderly persons who live at home. $N$ Engl J Med 2002;347:1068-74.

9. Stuck AE, Egger M, Hammer A, et al. Home visits to prevent nursing home admission and functional decline in elderly people: systematic review and meta-regression analysis. JAMA 2002;287:1022-8.

10. Pilotto A, Cella A, Pilotto A, et al. Three decades of comprehensive geriatric assessment: evidence coming from different healthcare settings and specific clinical conditions. J Am Med Dir Assoc 2017;18:192.e1-11.

11. Garrard JW, Cox NJ, Dodds RM, et al. Comprehensive geriatric assessment in primary care: a systematic review. Aging Clin Exp Res 2020;32:197-205.

12. Phelan EA, Balderson $B$, Levine $M$, et al. Delivering effective primary care to older adults: a randomized, controlled trial of the senior resource team at group health cooperative. J Am Geriatr Soc 2007;55:1748-56.

13. Barkhausen T, Junius-Walker U, Hummers-Pradier E, et al. "It's MAGIC" development of a manageable geriatric assessment for general practice use. BMC Fam Pract 2015;16:4.

14. Malmstrom TK, Voss VB, Cruz-Oliver DM, et al. The Rapid Cognitive Screen (RCS): a point-of-care screening for dementia and mild cognitive impairment. $J$ Nutr Health Aging 2015;19:741-4.

15. Senn N, Monod S. Development of a comprehensive approach for the early diagnosis of geriatric syndromes in general practice. Front Med (Lausanne) 2015;2:78

16. Vellas B, Balardy L, Gillette-Guyonnet S, et al. Looking for frailty in communitydwelling older persons: the Gérontopôle Frailty Screening Tool (GFST). J Nutr Health Aging 2013;17:629-31.

17. Blom J, den Elzen W, van Houwelingen AH, et al. Effectiveness and costeffectiveness of a proactive, goal-oriented, integrated care model in general practice for older people. A cluster randomised controlled trial: Integrated Systematic Care for older People - the ISCOPE study. Age Ageing 2016;45:30-41.

18. Ruikes FGH, Zuidema SU, Akkermans RP, et al. Multicomponent program to reduce functional decline in frail elderly people: a cluster controlled trial. J Am Board Fam Med 2016;29:209-17.

19. Integrated care for older people (ICOPE): guidance for person-centred assessment and pathways in primary care. Geneva: World Health Organization, 2019.

20. Macdonald SH-F, Travers J, Shé ÉN, et al. Primary care interventions to address physical frailty among community-dwelling adults aged 60 years or older: a meta-analysis. PLoS One 2020;15:e0228821.

21. Locatelli I, Monod S, Cornuz J, et al. A prospective study assessing agreement and reliability of a geriatric evaluation. BMC Geriatr 2017;17:153.

22. Mueller YK, Monod S, Locatelli I, et al. Performance of a brief geriatric evaluation compared to a comprehensive geriatric assessment for detection of geriatric syndromes in family medicine: a prospective diagnostic study. BMC Geriatr 2018;18:72.

23. Felix S, Guillemin P, Lleshi E, et al. Évaluation gériatrique globale. Geneva: Médecine et Hygiène; 2014:1-132.
24. Katz S, Ford AB, Moskowitz RW, et al. Studies of illness in the aged. the index of ADL: a standardized measure of biological and psychosocial function. JAMA 1963;185:914-9

25. Lawton MP, Brody EM. Assessment of older people: self-maintaining and instrumental activities of daily living. Gerontologist 1969;9:179-86.

26. Dalby DM, Sellors JW, Fraser FD, et al. Screening seniors for risk of functional decline: results of a survey in family practice. Can J Public Health 1999; 90:133-7.

27. Ciesla JR, Shi L, Stoskopf CH, et al. Reliability of Katz's activities of daily living scale when used in telephone interviews. Eval Health Prof 1993;16: 190-203.

28. Spector WD, Fleishman JA. Combining activities of daily living with instrumental activities of daily living to measure functional disability. J Gerontol B Psychol Sci Soc Sci 1998;53:S46-57.

29. Viret O, Schwarz J, Senn N, et al. Discussing age-related functional decline in family medicine: a qualitative study that explores both patient and physician perceptions. Age Ageing 2019;49:292-9.

30. Morley JE, Arai H, Cao L, et al. Integrated care: enhancing the role of the primary health care professional in preventing functional decline: a systematic review. J Am Med Dir Assoc 2017;18:489-94.

31. Counsell SR, Callahan CM, Clark DO, et al. Geriatric care management for lowincome seniors: a randomized controlled trial. JAMA 2007;298:2623-33.

32. Gil-Salcedo A, Dugravot A, Fayosse A, et al. Healthy behaviors at age 50 years and frailty at older ages in a 20-year follow-up of the UK Whitehall II cohort: a longitudinal study. PLoS Med 2020;17:e1003147.

33. Cohidon C, Cornuz J, Senn N. Primary care in Switzerland: evolution of physicians' profile and activities in twenty years (1993-2012). BMC Fam Pract 2015;16:107.

34. Grol SM, Molleman GRM, Kuijpers A, et al. The role of the general practitioner in multidisciplinary teams: a qualitative study in elderly care. BMC Fam Pract 2018;19:40.

35. Russell GM, Dahrouge S, Hogg W, et al. Managing chronic disease in ontario primary care: the impact of organizational factors. Ann Fam Med 2009; 309-18.

36. Sheehan C, Domingue BW, Crimmins E. Cohort trends in the gender distribution of household tasks in the United States and the implications for understanding disability. J Aging Health 2019;31:1748-69.

37. Salisbury C, Man M-S, Bower P, et al. Management of multimorbidity using a patient-centred care model: a pragmatic cluster-randomised trial of the 3D approach. Lancet 2018;392:41-50.

38. Glasgow RE, Whitesides H, Nelson CC, et al. Use of the Patient Assessment of Chronic Illness Care (PACIC) with diabetic patients: relationship to patient characteristics, receipt of care, and self-management. Diabetes Care 2005;28:2655-61.

39. Krucien N, Le Vaillant M, Pelletier-Fleury N. Adaptation and validation of the patient assessment of chronic illness care in the French context. BMC Health Serv Res 2014;14:269.

40. Joober H, Chouinard M-C, King J, et al. The patient experience of integrated care scale: a validation study among patients with chronic conditions seen in primary care. Int J Integr Care 2018;18:1.

41. Dolovich L, Oliver D, Lamarche L, et al. Combining volunteers and primary care teamwork to support health goals and needs of older adults: a pragmatic randomized controlled trial. CMAJ 2019;191:E491-500.

42. Black S, Nicholas C, Cotton S, et al. Determining discharge destination in geriatric evaluation and management units: Is progressive goal attainment a better early indicator of discharge destination than improvement in functional independence measure scores? Geriatr Gerontol Int 2018;18: 1058-63.

43. Giovannetti ER, Clair CA, Jennings LA, et al. Standardised approach to measuring goal-based outcomes among older disabled adults: results from a multisite pilot. BMJ Qual Saf 2021;30:157-66 
Competing interests: None declared.

This article has been peer reviewed.

Affiliations: University Centre for Primary care and Public Health (Unisanté) (Mueller, Schwarz, Locatelli, Senn) and Service of Geriatric Medicine and Geriatric Rehabilitation (Monod), Faculty of Biology and Medicine, University of Lausanne, Lausanne, Switzerland.

Contributors: Stéfanie Monod and Nicolas Senn conceived the intervention. Yolanda Mueller, Isabella Locatelli and Nicolas Senn designed the study. Yolanda Mueller and Nicolas Senn supervised data collection, and Yolanda Mueller, Joëlle Schwarz and Isabella Locatelli analyzed the data. All authors contributed to data interpretation. Yolanda Mueller wrote the first draft of the manuscript. All of the authors revised it critically for important intellectual content, gave final approval of the version to be published and agreed to be accountable for all aspects of the work.

Content licence: This is an Open Access article distributed in accordance with the terms of the Creative Commons Attribution (CC BY-NC-ND 4.0) licence, which permits use, distribution and reproduction in any medium, provided that the original publication is properly cited, the use is noncommercial (i.e., research or educational use), and no modifications or adaptations are made. See: https://creativecommons.org/licenses/by-nc-nd/4.0/
Funding: The trial was funded by the Swiss National Science Foundation Grant 32003B_159863/1 until February 2019. Trial completion was self-funded.

Data sharing: The supporting data for this article are available on request on the Unisanté data repository: https://doi.org/10.16909/ dataset/23.

Disclaimer: The funder had no role in study design; in the collection, analysis, and interpretation of data; in the writing of the report; and in the decision to submit the article for publication. All authors had full access to all of the data in the study and can take responsibility for the integrity of the data and the accuracy of the data analysis.

Acknowledgements: The authors thank all participating general practitioners (GPs) and patients, and all geriatricians who reviewed the AGE recommendations and participated in GP training. The authors also thank Dr. Ophélie Viret for her contribution to study implementation and acquisition of the data, Prof. Jacques Cornuz and Prof. Christophe Büla for their input into the study conception. The authors thank the GPs of the pilot committee for guiding the case report forms and study procedures, and the study staff for data collection and monitoring. The authors thank Melanie Price Hirt for English language editing.

Accepted: June 1, 2021

Correspondence to: Yolanda Mueller, Yolanda.mueller@unisante.ch 